\title{
Epileptic seizures in multiple sclerosis: prevalence, competing causes and diagnostic accuracy
}

\author{
Friederike Neuß ${ }^{1} \cdot$ Felix von Podewils ${ }^{1}\left[\right.$ D $\cdot$ Zhong Irene Wang $^{2} \cdot$ Marie Süße $^{1}\left(\mathbb{D} \cdot\right.$ Uwe Klaus Zettl $^{3}$. \\ Matthias Grothe ${ }^{1}$ (1)
}

Received: 23 October 2020 / Revised: 22 November 2020 / Accepted: 4 December 2020 / Published online: 15 December 2020

(c) The Author(s) 2020

\begin{abstract}
Background Multiple sclerosis (MS) is accompanied by an increased risk of epileptic seizures, but data with a detailed description of the competing causes are lacking.

Methods We aimed to describe a cohort of patients with both MS and epileptic seizures in a retrospective, population-based study.

Results We included 59 out of 2285 MS patients who had at least one epileptic seizure. Out of them, 22 had seizures before the diagnosis of MS, whereas epileptic seizures occurred after MS diagnosis in 37 patients, resulting in a total prevalence of epileptic seizures in MS of 2.6\%. Competing causes could be found in 50.8\% (30/59) of all patients, with 40.9\% (9/22) compared to $56.8 \%$ (21/37) of the MS patients with seizures before vs after MS diagnosis. The main alternative causes were traumatic brain injury and cerebral ischemia accounting for more than $30 \%$ of the patients, with no difference between the subgroups. $33.3 \%$ and $55.6 \%$ of MS patients with seizures before/after MS diagnosis had documented pathological EEG alterations.

Conclusion A remarkable percentage of MS patients with epileptic seizures do have alternative competing causes at the time of the first seizure. A detailed diagnostic setup including patient history, EEG and MRI is recommended in the evaluation and choice for the best treatment.
\end{abstract}

Keyword Multiple sclerosis $\cdot$ Epilepsy $\cdot$ Seizures $\cdot$ Comorbidity $\cdot$ Epidemiology

\section{Introduction}

The association between multiple sclerosis (MS) and epileptic seizures has been described for more than 30 years [1]. Several recent studies confirmed a threefold increased risk for epileptic seizures in MS patients compared to healthy controls [2-5]. Seizures can occur as first symptom in MS

Uwe Klaus Zettl and Matthias Grothe contributed equally to this work.

Matthias Grothe

matthias.grothe@med.uni-greifswald.de

1 Department of Neurology, University Medicine Greifswald, Ferdinand-Sauerbruchstraße, 17475 Greifswald, Germany

2 Epilepsy Center, Neurological Institute, Cleveland Clinic Foundation, Cleveland, OH, USA

3 Department of Neurology, Neuroimmunological Section, University of Rostock, Rostock, Germany
[6-8], but the cumulative incidence rises with disease duration up to nearly $6 \%$ [2].

The underlying cause of epileptic seizures in MS is not well understood. Imaging studies in small samples of well characterized MS patients suggest grey matter pathology particularly in the temporal lobes to be associated with a higher risk of epilepsy [9-11].

On the other hand, seizures can be caused by several other brain pathologies such as traumatic injury, infection, neoplasia, or stroke [12-14], which can also occur in MS, especially with increasing disease duration [15]. In a very recent study, the rate of epilepsy in MS was much lower after excluding all alternative etiologies, suggesting that the causes of epilepsy in MS might be heterogeneous [16].

Another important issue is the diagnostic accuracy. Detailed information on how the diagnosis of epilepsy was initially made is often not available, especially in registry studies. According to the guidelines of the International League Against Epilepsy (ILAE), diagnosis of epilepsy can 
be made after two unprovoked seizures occurring more than $24 \mathrm{~h}$ apart, after one unprovoked seizure with a high recurrence risk or after diagnosis of an epilepsy syndrome [17, 18]. Apart from clinical appearance of seizures, electroencephalogram (EEG), especially in combination with provocation methods, is seen as an important tool to differentiate between epileptic and non-epileptic seizures [19]. Existing data suggest that up to $70 \%$ of patients with epilepsy are misdiagnosed, highlighting the importance of a detailed clinical assessment and EEG in the diagnostic process [20].

Taken together, the literature about epilepsy in MS is rather insufficient. The aim of this retrospective study, therefore, was to characterize a cohort of MS patients with known epileptic seizures more precisely with respect to competing risk factors, onset of seizures in relation to MS onset, as well as diagnostic procedures and accuracy. To investigate the effect of comorbidities on the relationship between epilepsy and MS, we further dichotomized the cohort in two subgroups, one with MS diagnosed after the onset of seizures (S-MS) and another with seizures occurring after the diagnosis of MS (MS-S).

\section{Materials and methods}

The retrospective population-based cohort study was approved by the local ethics committee of the University medicine Greifswald (BB022/19). We screened medical reports from the MS outpatient clinics from the departments of Neurology, University medicine Greifswald and University medicine Rostock, for patients who have been treated in at least one of these two specialized centers between 03/2009 and 03/2019. Both hospitals offer specialized MS treatment in the entire catchment area of about 750,000 people.

Clinical data were collected by reviewing the patients' medical records and included age, gender, disease course of both MS as well as epileptic seizures and/or epilepsy, including progression in time, treatment patterns and severity. Comorbidities were classified in categories according to ICD-10 codes [21]. Patients were excluded if medical reports were incomplete, patients did not fulfill diagnostic criteria for MS [22, 23], or the characterization of seizures was questionable. All variables were collected at the time of the first seizure as well as at the time of the last reported visit.

SPSS 21 (IBM Co., Armonk, New York, USA) was used for statistical processing of the data. For the group statistics for the competing causes, Fishers exact test was computed with the significance level set at $p<0.05$.

\section{Results}

\section{Selection of cohort}

Between 2009 and 2019, a total of 2285 MS patients were treated in the two university outpatient clinics in Rostock and Greifswald. Out of these, a group of $61 \mathrm{MS}$ patients was defined with at least one epileptic seizure. Two patients were excluded due to insufficient documentation. Therefore, our final cohort consists of 59 patients, resulting in a prevalence of $2.6 \%$ (Fig. 1).
Fig. 1 Flowchart summarizing the selection of patients that constitute our final cohort
MS-patients treated in two outpatient clinics between 2009 and 2019 $(n=2285)$

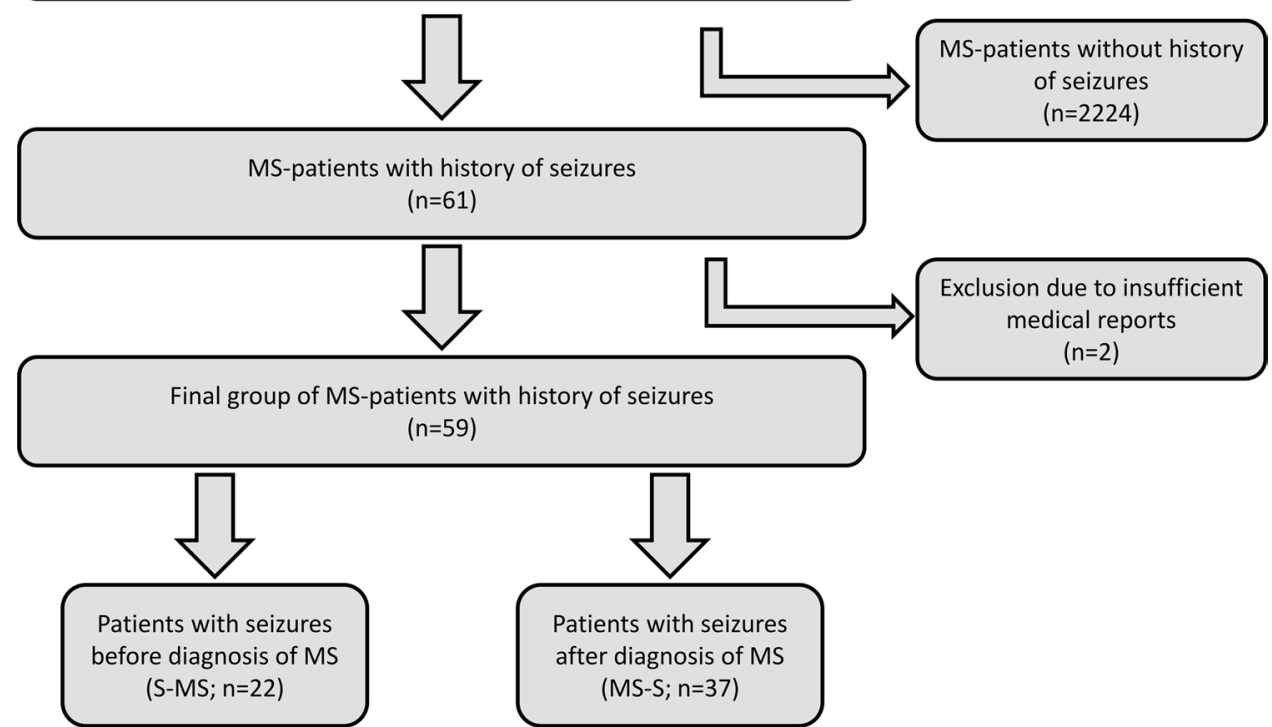


Table 1 Demographical and clinical data

\begin{tabular}{llll}
\hline & $\begin{array}{l}\text { All MS } \\
\text { patients } \\
(n=59)\end{array}$ & $\begin{array}{l}\text { S-MS } \\
(n=22, \\
37.3 \%)\end{array}$ & $\begin{array}{l}\text { MS-S } \\
(n=37, \\
62.7 \%)\end{array}$ \\
\hline Age (years/mean) & 51.7 & 46.2 & 55.0 \\
$\begin{array}{l}\text { EDSS (median) } \\
\text { Disease duration at time of } \\
\text { last reported visit (month/ } \\
\text { mean) }\end{array}$ & 5.0 & 3.0 & 6.0 \\
$\begin{array}{l}\text { Disease course at time of last reported visit }(n, \% \text { of patients in } \\
\text { group) }\end{array}$ & 98.3 & 232.5 \\
RRMS & $31,52.5$ & $17,77.3$ & $14,37.8$ \\
SPMS & $25,42.4$ & $4,18.2$ & $21,56.8$ \\
PPMS & $3,5.1$ & $1,4.5$ & $2,5.4$ \\
\hline
\end{tabular}

Table 2 Antiepileptic treatment at time of last visit (patients with more than one medication; ${ }^{\text {ain two patients; }}{ }^{\text {bin one patient each) }}$

\begin{tabular}{|c|c|c|}
\hline \multicolumn{3}{|c|}{ Patients with double-therapy } \\
\hline Lamotrigine & Lacosamide & \\
\hline Lamotrigine & Mesuximide & \\
\hline Lamotrigine & Valproate & \\
\hline Levetiracetam & Clobazame & \\
\hline Levetiracetam & Lacosamide & \\
\hline Levetiracetam & Oxcarbazepine & \\
\hline Oxcarbazepine & Gabapentin & \\
\hline Valproate & Carbamazepine & \\
\hline \multicolumn{3}{|c|}{ Patients with triple-therapy } \\
\hline Lamotrigine & Levetiracetam & Eslicarbazepinacetate \\
\hline Lamotrigine & Levetiracetam & Lacosamide \\
\hline
\end{tabular}

\section{Demographical and clinical data}

Female patients consisted of 76.3\% (45/59) of all patients. Mean age at last reported visit was 51.7 years (SD 13.6), median EDSS 5.0 (QR 2.0-6.5). At time of last reported visit, $52.5 \%$ (31/59) had relapsing-remitting disease course (RRMS), 42.4\% (25/59) had secondary-progressive MS (SPMS) and 5.1\% (3/59) primary-progressive MS (PPMS) (see Table 1).

The most frequently used anti-seizure drugs (ASD) at last reported visit was levetiracetam $(32.2 \%, 19 / 59)$, lamotrigin $(25.4 \%, 15 / 59)$, valproate $(10.2 \%, 6 / 59)$, and carbamazepin $(8.5 \%, 5 / 59)$. Nine patients out of $59(15 \%)$ were treated with a combination of two ASD, two (3\%) with a combination of three ASD (see Table 2), whereas 27.1\% (16/59) of patients had no ASD.

According to the available medical reports, $41.8 \%$ (23/55) of the MS patients only had one single epileptic seizure. Of those with recurrent seizures $32.7 \%$ (18/55) had not more than one seizure per year and $25.5 \%(14 / 55)$ had one or more seizures per year.
At the time of the first seizure, $26.8 \%(15 / 56)$ of the patients received disease-modifying-treatment (DMT) for MS, consisting of interferon-beta $(14.3 \%, 8 / 56)$, glatiramer acetat $(3.6 \%, 2 / 56)$, dimethylfumarat $(1.8 \%, 1 / 56)$, fingolimod $(1.8 \%, 1 / 56)$, mitoxantron $(1.8 \%, 1 / 56)$, natalizumab $(1.8 \%, 1 / 56)$, methotrexat $(1.8 \%, 1 / 56)$. Three patients had to be excluded for this analysis due to missing information on the DMT.

At the time of the last documented visit, pharmacological treatment of MS consisted of fingolimod (15.3\%, 9/59), interferon-beta $(13.6 \%, 8 / 59)$, glatiramer acetat $(6.8 \%, 4 / 59)$, dimethylfumarat $(3.4 \%, 2 / 59)$, mitoxantron $(1.7 \%, 1 / 59)$ or natalizumab $(1.7 \%, 1 / 59) .57 .6 \%$ (34/59) of the patients had no disease modifying therapy on last recorded visit.

\section{Diagnostic accuracy of epilepsy}

Due to the retrospective design and documentary weaknesses, no consistent information on the epilepsy classification is available.

Approximately half of the epileptic seizures were witnessed by neurologists $(14.0 \%, 8 / 57)$ or other physicians $(28.1 \%, 16 / 57)$, but the majority of the seizures $(57.9 \%$, 33/57) were only documented according to observations from non-medical professionals. Two patients had to be excluded for this analysis due to missing information about seizure documentation.

Information about EEG could be obtained in 48 out of 59 patients, which always was routine EEG. In 58.3\% (28/48) of these patients, pathological EEG alterations (regional or generalized slowing, focal or generalized epileptiform discharges) were documented, whereas $41.7 \%$ (20/48) had normal EEG findings.

In total, one-third of the seizures $(33.3 \%, 16 / 48)$ were diagnosed and classified only according to patient documentation (i.e. without pathological EEG and without direct observation).

\section{Competing causes of epilepsy}

In our cohort of 59 patients, $30(50.8 \%)$ had risk factors documented in the medical reports that can cause epileptic seizures and can therefore be an alternative cause, such as traumatic brain injury $(18.6 \%, 11 / 59)$, cerebral ischemia $(10.2 \%, 6 / 59)$, migraine $(n=5,8.5 \%)$, brain tumor $(n=3$, $5.1 \%)$, and drug abuse $(n=3,5.1 \%)$ (details in Table 3 ).

\section{Group comparison S-MS vs. MS-S}

A total of 37 patients (62.7\%) showed seizures after the diagnosis of MS and were categorized into the MS-S subgroup; 22 patients $(37.3 \%)$ showed seizures before MS was diagnosed and were categorized into the S-MS subgroup. 
Table 3 Competing causes for seizures and p-values for group comparison

\begin{tabular}{lllll}
\hline Risk factor & $\begin{array}{l}\text { Total }(n, \% \text { of } \\
\text { patients })\end{array}$ & $\begin{array}{l}\text { MS-S }(n, \% \text { of } \\
\text { patients })\end{array}$ & $\begin{array}{l}\text { S-MS }(n, \% \text { of } \\
\text { patients })\end{array}$ & $p$ \\
\hline$n=$ & 59 & 37 & 22 & $13,59.1 \%$ \\
None & $29,49.2 \%$ & $16,43.2 \%$ & $5,22.7 \%$ & 0.48 \\
Traumatic brain injury & $12,20.3 \%$ & $7,18.9 \%$ & $2,9.1 \%$ & 1 \\
Cerebral ischemia & $6,10.2 \%$ & $4,10.8 \%$ & $2,9.1 \%$ & 1 \\
Migraine & $5,8.5 \%$ & $3,8.1 \%$ & $0,0 \%$ & 0.54 \\
Brain tumor & $3,5.1 \%$ & $3,8.1 \%$ & $0,0 \%$ & 0.54 \\
Drug abuse & $3,5.1 \%$ & $3,8.1 \%$ & $0,0 \%$ & 0.53 \\
Intracranial hemorrhage & $2,3.4 \%$ & $2,5.4 \%$ & $1,4.5 \%$ & 1 \\
Infantile brain damage & $2,3.4 \%$ & $1,2.7 \%$ & $0,0 \%$ & 0.53 \\
Meningioma & $2,3.4 \%$ & $2,5.4 \%$ & $0,0 \%$ & 0.53 \\
Meningitis & $2,3.4 \%$ & $2,5.4 \%$ & $1,4.5 \%$ & 1 \\
Psychological trauma & $2,3.4 \%$ & $1,2.7 \%$ & $0,0 \%$ & 1 \\
Alcohol abuse & $1,1.7 \%$ & $1,2.7 \%$ & $0,0 \%$ & 1 \\
Brain abscess & $1,1.7 \%$ & $1,2.7 \%$ & $1,4.5 \%$ & 0.36 \\
Developmental venous anomalies & $1,1.7 \%$ & $0,0 \%$ & $1,4.5 \%$ & 0.36 \\
Encephalitis & $1,1.7 \%$ & $0,0 \%$ & $0,0 \%$ & 1 \\
Epidural hematoma & $1,1.7 \%$ & $1,2.7 \%$ & $1,4.5 \%$ & 0.36 \\
Sinus vein thrombosis & $1,1.7 \%$ & $0,0 \%$ & $0,0 \%$ & 1 \\
Stereotactic biopsy & $1,1.7 \%$ & $1,2.7 \%$ & $0,0 \%$ & 1 \\
Stereotactic deep brain stimulation & $1,1.7 \%$ & $1,2.7 \%$ & $1,4.5 \%$ & 0.36 \\
Subdural hematoma & $1,1.7 \%$ & $0,0 \%$ & $0,0 \%$ & 1 \\
Subdural hygroma & $1,1.7 \%$ & $1,2.7 \%$ & & \\
\hline & & & & \\
\hline
\end{tabular}

In the MS-S subgroup, clinical course at the time of the first epileptic seizure was RRMS in 16/37 (43.2\%), SPMS in 19/37 (51.4\%) and PPMS in 2/37 (5.4\%).

Clinical course at the last recorded visit in MS-S was SPMS $(56.8 \%, 21 / 37)$, RRMS in 14 patients $(37.8 \%)$ and PPMS in 2 patients (5.4\%); in S-MS RRMS (17 patients, $77.3 \%)$, SPMS in 4 patients $(18.2 \%)$ and PPMS in one patient $(4.5 \%)$.

\section{Diagnostic accuracy of epilepsy between S-MS and MS-S}

In S-MS, 55.6\% of patients (10/18) had documented pathological EEG alterations. In contrast, in MS-S only 33.3\% (10/30) had EEG alterations.

Diagnosis of epilepsy was made only according to patient documentation in $28.6 \%(6 / 21) \mathrm{S}-\mathrm{MS}$ patients and in 11 out of $36(30.6 \%)$ patients of MS-S.

\section{Competing causes of epilepsy between S-MS and MS-S}

Although not significant $(p>0.25)$, patients with MS-S more frequently showed at least one additional risk factor for epileptic seizures in their patient history (21/37 patients; $56.8 \%$ ) compared to those with S-MS, where only $40.9 \%$ (9/22 patients) showed at least one competing cause. The most frequent risk factors were traumatic brain injury
(MS-S: $18.9 \%$ vs. S-MS: $22.7 \%)$, cerebral infarction $(10.8 \%$ vs. $9.1 \%)$, migraine $(8.1 \%$ vs. $9.1 \%)$, brain tumor $(8.1 \%$ vs. $0 \%)$ and drug abuse $(8.1 \%$ vs. $0 \%)$. Table 3 shows a complete list of competing causes.

\section{Discussion}

The goal of this retrospective population-based study was to investigate in detail a cohort of MS patients with epileptic seizures or the diagnosis of epilepsy to better characterize patients' demography, diagnostic accuracy, the onset of seizures in relation to MS onset as well as competing risk factors. For this purpose, we retrospectively analyzed medical reports of two large university outpatient clinics specialized in MS from the past 10 years. To our knowledge, this is the first study to systematically investigate MS and epileptic seizures in a population-based approach.

\section{Prevalence}

We showed the prevalence of epileptic seizures in MS is $2.6 \%$, which is in line with existing literature $[2,4]$ and higher compared to the general population [24]. Interestingly, in a recent study, the prevalence of epilepsy in MS was $0.9 \%$ using rather strict diagnostic criteria excluding patients 
with alternative etiologies [16]. Our data are also in line with this prevalence, as only $1.3 \%$ of all MS patients of our cohort had epileptic seizures with strict diagnostic criteria without any competing causes.

\section{Demographic and disease related characteristics}

The mean age in our cohort was 51.7 years at the last visit, predominantly women, which is comparable to existing cohort and registry studies [2, 25]. A systematic review by Gasparini et al. [26] revealed no difference between relapsing and progressive courses, whereas Burman and Zelano showed a risk increase of epilepsy by the factor 2.5 [2] in progressive disease courses. This seeming contradiction is in line with our findings that MS patients with epilepsy were equally distributed between relapsing and progressive disease forms, but differed between the subgroups S-MS and MS-S. In our cohort, $37.3 \%$ had the first seizure before the diagnosis of MS was made. Of this subgroup, 77.3\% of patients had a relapsing course at the time of the last visit. On the other hand, the most frequent course at the first epileptic seizure in MS-S was SPMS (51.4\%), with 56.8\% on the last reported visit, emphasizing the association with increasing disease duration in this subgroup. Additionally, we here describe a cohort of MS patients with seizures. As the vast majority (75\%) of our patients treated in the outpatient clinics were relapsing remitting, the ratio is much higher in progressive patients as well.

In our cohort, a high proportion of more than $50 \%$ of the patients with MS did not have any disease modifying drug at the last documented visit, which could essentially be due to the high percentage of secondary-progressive MS patients. Because the choice of treatment depends on the year and the country of the data collection, study comparison is difficult. In a Swedish registry study in 2017, almost $15 \%$ of the MS patients did not have any MS treatment. However, the lower proportion of patients with SPMS in this registry [2] might explain the difference from our data. Time of data collection, national guidelines, time of drug approval as well as local preferences can also explain differences in the choice of ASD. In our cohort, levetiracetam and lamotrigin were the most frequently used ASD in almost half of our patients, whereas in a Norwegian study the majority of patients were treated with carbamazepine [25]. In total, antiepileptic treatment was found in $72.9 \%$ of our patients, which is comparable to existing data from MS registries [27].

\section{Diagnostic accuracy}

To our knowledge, diagnostic accuracy of epileptic seizures and epilepsy in MS has not been investigated yet. According to the ILAE guidelines, diagnosis of epilepsy can be made after two unprovoked seizures occurring more than
$24 \mathrm{~h}$ apart [17]. However, in MS, the ILAE guidelines may be more difficult to interpret in cases of only one unprovoked seizure, as the recurrence risk mainly depends on the detection of an MRI lesion responsible for the seizure [17]. Although the diagnosis of MS largely depends on the evidence of characteristic lesions in the cerebral MRI, there is a diagnostic uncertainty whether these lesions actually are relevant to a seizure onset zone, especially as cortical lesions are difficult to detect in routine imaging [28]. According to the ILAE classification, structural etiology “....refers to abnormalities visible on structural neuroimaging where the electro-clinical assessment together with the imaging findings lead to a reasonable inference that the imaging abnormality is the likely cause of the patient's seizure..." [18]. It can be assumed that in a considerable proportion of MS patients, the diagnosis of structural epilepsy was perhaps made prematurely, since this prerequisite is difficult to prove.

Interestingly, the interictal EEG was pathologic in onethird of the MS-S subgroup and more than 50\% in the S-MS subgroup. In the existing case series, pathological EEG was highly prevalent (13 of 13 patients in [25], 12 of 14 patients in [24]), but the inclusion criteria for these studies were different compared to the current study with our populationbased approach.

Nevertheless, in one-third of our patients, epileptic seizures or even epilepsy was diagnosed only on history. Taken this into account and considering the very high rate of pathologic EEG findings in MS patients shown here, a detailed and well thought-out diagnostic scheme including EEG diagnostic is strongly recommended to further strengthen the diagnosis of epilepsy in MS and to exclude competing causes for seizures.

\section{Onset epilepsy in relation to MS}

In 22 of 59 (37.3\%) patients with MS and epilepsy, the first epileptic seizure occurred before the diagnosis of MS was made. Compared to other studies [6], this large proportion in our cohort was rather surprising. An association between the epileptic seizures and the later diagnosed MS cannot be clearly proven. However, none of these patients was diagnosed with epilepsy syndromes such as genetic generalized epilepsy. On the other hand, possible competing risk factors for epileptic seizures were found in nearly $40 \%$ of the patients in the subgroup of S-MS patients, most frequent traumatic brain injury. Taken this into account, one can hypothesize that early subtle and clinically irrelevant cortical lesions in the later manifest MS might already have caused the seizures.

There is a long debate if epileptic seizures might be the initial clinical manifestation of MS, and the existing literature (summarized and discussed in [29]) as well as our cohort study suggest that seizures can occur before the 
diagnosis MS is made. Some case series also suggest seizures occurring predominantly during relapses, or as the only clinical sign of a relapse [30]. In our cohort, we did not find any of such cases, although we cannot rule out the possibility of incomplete medical reports.

As this retrospective study only analyses existing medical reports, we did not have detailed information about the diagnostic that was made during the time the seizure occurred. Further prospective studies have to systematically investigate MS-related pathology like temporal cortical lesions that might lead to epilepsy in MS $[10,11]$.

\section{Competing risk factors}

Alternative causes for epileptic seizures were highly frequent, seen in half of our cohort with a vast majority of structural etiologies. In several other studies, patients with competing risk factors have been excluded from further analysis $[6,16]$. In a very recent study on epilepsy in multiple sclerosis, Langenbruch et al. excluded 16 out of 90 patients for further analysis because of other potential risk factors for epilepsy besides MS [16]. In a French cohort, 35 out of 102 patients with MS and epilepsy had documented alternative risk factors, mainly associated with not only early onset of the seizures in infancy (24 out of 35) [6], but also cerebral haemorrhage or tumor. In contrast, we aimed to document all potential competing risk factors for epilepsy in MS patients, which theoretically explains half the prevalence of our cohort. We are aware of the different epileptogenic risk of different underlying conditions; for example, brain tumor or intracranial hemorrhage are associated with a higher risk to cause epileptic seizures compared to migraine [31]. However, the basic idea of this study was to describe the risk of epileptic seizures and epilepsy in MS in a real-life approach.

Seizures always can be provoked by various pathologies other than MS such as traumatic injury, infection, neoplasia or stroke. According to a prospective study from Iceland, $9 \%$ of unprovoked seizures in adults are attributable to brain trauma, 7\% to cerebrovascular disease [14], a Swedish epilepsy registry describes brain tumor (ca. 4\%) and stroke (ca. $5 \%)$ as the main presumed etiologies of first seizures [32]. In our study, trauma (20\%) and cerebral ischemia (10\%) had been the main alternative causes as well, and brain tumor also identifiable in $5 \%$ of our patients. As these competing risk factors are descriptive, no further etiological allocation is possible.

Interestingly, no difference was seen with respect to risk factors between S-MS and MS-S. This can be interpreted as a systemically proportion of other CSN pathology leading to seizures. On the other hand, it might also be a hint that seizures may be part of MS, as there is no systematic difference in alternative causes.

\section{Limitations}

Since our research has been carried out retrospectively and is based on patient documentation, in some cases the information collected was different, incomplete or in some cases missing altogether. For example, the type of epileptic seizures was rarely documented, which is why we dispensed with the evaluation completely. Additionally, the retrospective design and the inclusion of patients treated at specialized tertiary referral centers might be a source of a selection bias. Despite these limitations, this population-based approach for the first time ever gives a rather detailed overview about MS patients with epilepsy.

\section{Conclusion}

Seizures in patients with MS are more frequent than in general population, although common assumptions on prevalence rates might be overestimated. Our real-life data on MS patients with epileptic seizures show that competing risk factors can be found in up to $50 \%$ of the patients and are comparable to other patients with structural epilepsy. However, the decision whether to start ASD treatment or not is largely dependent on the estimation of the risk of seizure recurrence. Therefore, to prove the association between seizures and MS and to exclude other etiologies as underlying causes, it is important to have detailed information about competing risk factors and to perform a targeted diagnostic including EEG and MRI.

Author contributions UKZ and MG contributed to the study conception and design. Material preparation, data collection and analysis were performed by FN. The first draft of the manuscript was written by FN and all authors commented on previous versions of the manuscript. All authors read and approved the final manuscript.

Funding Open Access funding enabled and organized by Projekt DEAL.. This research received no specific Grant from any funding agency.

\section{Compliance with ethical standards}

Conflicts of interest F.N. declares no financial or other conflicts of interest. F.v.P. obtained honoraria for speaking engagements from Desitin, EISAI, UCB, BIAL and GW Pharma and was part of speakers's bureau of Desitin, EISAI, UCB, BIAL and GW Pharma. Z.I.W. declares no financial or other conflicts of interest. M.S. declares no financial or other conflicts of interest. U.K.Z. received honoraria and travel reimbursements for attending meetings from Alexion, Almirall, Bayer Health Care, Celgene, Sanofi Genzyme, Merck Serono, Novartis, Roche, TEVA. His research is funded by German Ministry for Education and Research (BMBF), German Ministry for Economy (BMWi), Deutsche Forschungsgemeinschaft (DFG) and European Union (EU). M.G. received honoraria and travel reimbursements for attending meetings from Biogen, Celgene, Merck Serono, Novartis, Roche, Sa- 
nofi Genzyme, TEVA. His research is funded by German Ministry for Education and Research (BMBF), Merck Serono and Novartis.

Ethics approval The data collection for this study was approved by the local ethics committee of the University Medicine Greifswald (BB022/19).

Open Access This article is licensed under a Creative Commons Attribution 4.0 International License, which permits use, sharing, adaptation, distribution and reproduction in any medium or format, as long as you give appropriate credit to the original author(s) and the source, provide a link to the Creative Commons licence, and indicate if changes were made. The images or other third party material in this article are included in the article's Creative Commons licence, unless indicated otherwise in a credit line to the material. If material is not included in the article's Creative Commons licence and your intended use is not permitted by statutory regulation or exceeds the permitted use, you will need to obtain permission directly from the copyright holder. To view a copy of this licence, visit http://creativecommons.org/licenses/by/4.0/.

\section{References}

1. Kinnunen E, Wikström J (1986) Prevalence and prognosis of epilepsy in patients with multiple sclerosis. Epilepsia 27:729-733

2. Burman J, Zelano J (2017) Epilepsy in multiple sclerosis: a nationwide population-based register study. Neurology 89:2462-2468

3. Allen AN, Seminog OO, Goldacre MJ (2013) Association between multiple sclerosis and epilepsy: large population-based recordlinkage studies. BMC Neurol 13:189

4. Marrie RA, Reider N, Cohen J et al (2015) A systematic review of the incidence and prevalence of sleep disorders and seizure disorders in multiple sclerosis. Mult Scler 21:342-349

5. Olafsson E, Benedikz J, Hauser WA (1999) Risk of epilepsy in patients with multiple sclerosis: a population-based study in Iceland. Epilepsia 40:745-747

6. Catenoix H, Marignier R, Ritleng C et al (2011) Multiple sclerosis and epileptic seizures. Mult Scler 17:96-102

7. Spatt J, Chaix R, Mamoli B (2001) Epileptic and non-epileptic seizures in multiple sclerosis. J Neurol 248:2-9

8. Kinnunen E (1984) Multiple sclerosis in Finland: evidence of increasing frequency and uneven geographic distribution. Neurology 34:457-461

9. Calabrese M, Castellaro M, Bertoldo A et al (2017) Epilepsy in multiple sclerosis: the role of temporal lobe damage. Mult Scler 23:473-482

10. Calabrese M, Grossi P, Favaretto A et al (2012) Cortical pathology in multiple sclerosis patients with epilepsy: a 3 year longitudinal study. J Neurol Neurosurg Psychiatry 83:49-54

11. Calabrese M, de Stefano N, Atzori M et al (2008) Extensive cortical inflammation is associated with epilepsy in multiple sclerosis. J Neurol 255:581-586

12. Syvertsen M, Nakken KO, Edland A et al (2015) Prevalence and etiology of epilepsy in a Norwegian county-a population based study. Epilepsia 56:699-706

13. Vezzani A, Aronica E, Mazarati A et al (2013) Epilepsy and brain inflammation. Exp Neurol 244:11-21
14. Olafsson E, Ludvigsson P, Gudmundsson G et al (2005) Incidence of unprovoked seizures and epilepsy in Iceland and assessment of the epilepsy syndrome classification: a prospective study. Lancet Neurol 4:627-634

15. Sanai SA, Saini V, Benedict RH et al (2016) Aging and multiple sclerosis. Mult Scler 22:717-725

16. Langenbruch L, Krämer J, Güler S et al (2019) Seizures and epilepsy in multiple sclerosis: epidemiology and prognosis in a large tertiary referral center. J Neurol 266:1789-1795

17. Fisher RS, Acevedo C, Arzimanoglou A et al (2014) ILAE official report: a practical clinical definition of epilepsy. Epilepsia $55: 475-482$

18. Scheffer IE, Berkovic S, Capovilla $Z$ et al (2017) ILAE classification of the epilepsies: position paper of the ILAE commission for classification and terminology. Epilepsia 58:512-521

19. Dericioğlu N, Saygi S, Ciğger A (1999) The value of provocation methods in patients suspected of having non-epileptic seizures. Seizure 8:152-156

20. Xu Y, Nguyen D, Mohamed A et al (2016) Frequency of a false positive diagnosis of epilepsy: a systematic review of observational studies. Seizure 41:167-174

21. World Health Organization (2004) ICD-10: International statistical classification of diseases and related health problems. 10th revision, 2nd ed.

22. Polman CH, Reingold SC, Banwell B et al (2011) Diagnostic criteria for multiple sclerosis: 2010 revisions to the McDonald criteria. Ann Neurol 69:292-302

23. Thompson AJ, Banwell BL, Barkhof F et al (2018) Diagnosis of multiple sclerosis: 2017 revisions of the McDonald criteria. Lancet Neurol 17:162-173

24. Fiest KM, Sauro KM, Wiebe S et al (2017) Prevalence and incidence of epilepsy: a systematic review and meta-analysis of international studies. Neurology 88:296-303

25. Benjaminsen E, Myhr K-M, Alstadhaug KB (2017) The prevalence and characteristics of epilepsy in patients with multiple sclerosis in Nordland county. Norway Seizure 52:131-135

26. Gasparini S, Ferlazzo E, Ascoli M et al (2017) Risk factors for unprovoked epileptic seizures in multiple sclerosis: a systematic review and meta-analysis. Neurol Sci 38:399-406

27. Rommer PS, Eichstadt K, Ellenberger D et al (2019) Symptomatology and symptomatic treatment in multiple sclerosis: results from a nationwide MS registry. Mult Scler 25:1641-1652

28. Filippi M, Preziosa P, Banwell BL et al (2019) Assessment of lesions on magnetic resonance imaging in multiple sclerosis: practical guidelines. Brain 142:1858-1875

29. Poser CM, Brinar VV (2003) Epilepsy and multiple sclerosis. Epilepsy Behav 4:6-12

30. Sokic DV, Stojsavljevic N, Drulovic J et al (2001) Seizures in multiple sclerosis. Epilepsia 42:72-79

31. Nye BL, Thadani VM (2015) Migraine and epilepsy: review of the literature. Headache 55:359-380

32. Adelöw C, Andell E, Amark P et al (2009) Newly diagnosed single unprovoked seizures and epilepsy in Stockholm, Sweden: first report from the Stockholm Incidence Registry of Epilepsy (SIRE). Epilepsia 50:1094-1101 